\title{
Progressive Focal Sclerosis of Retinal Arteries: a Sequel to Impaction of Cholesterol Emboli
}

\author{
A. J. DARK, M.D., F.R.C.S.ED., D.o. ; S. N. RIZK, † M.B., CH.B., F.R.C.S., D.o.
}

[With Special Plate]

Brit. med. F., 1967, 1, 270-273

Bright plaques, usually situated at bifurcations of retinal arteries and arterioles, have long been regarded as local arteriosclerotic manifestations. Such plaques, which are gleaming white or coppery in colour, may exhibit scintillation (especially when extraneous pressure is exerted on the globe), flashing synchronously with the pulse or when the incident ophthalmoscopic light strikes them at an appropriate angle. They sometimes seem to be wider than the vessel in which they lie, giving the impression that they are located intramurally rather than in the lumen. This impression, coupled with the observation that total occlusion of the lumen occurs only exceptionally, doubtless influenced previous observers to consider them as atheromatous plaques which had developed in situ.

In sharp contrast to this classical view the work of Hollenhorst $(1961,1962)$ has provided good evidence for the embolic nature of such plaques. According to him such plaques are thought to originate from eroded atheromata involving the carotid arteries, the heart valves, or the aorta, reaching the retinal arteries as blood-borne emboli. Hollenhorst noted that these plaques occurred in the ipsilateral retinal arteries in $11 \%$ of the 235 cases of carotid occlusive disease, whereas they were noted in only $4 \%$ of 93 cases of vertebrobasilar insufficiency. They were interpreted as thin lamellae of crystalline material, possibly consisting of or containing cholesterol.

Necropsy findings have directed several writers (Flory, 1945 ; Handler, 1956 ; Sayre and Campbell, 1959) to postulate that cholesterol emboli originating from eroded atheromatous aortic sites had lodged in small arteries of 50 to $900 \mu$ diameter in kidney, heart, pancreas, thyroid, and cerebrum. Microscopical study revealed cholesterol crystals embedded in hyperplastic intimae. The significance of these appearances has not been easy to interpret, because deposition of cholesterol as a concomitant of local arteriosclerotic change, in contradistinction to its embolic impaction, could not be excluded.

In the present study two patients presenting with transient uniocular loss of vision and showing glistening plaques in the retinal arteries were studied ophthalmoscopically with frequent photographic recording of the appearances over many months. It is thought that these findings provide further evidence of the embolic nature of such plaques. Subsequent development of changes in the arterial walls adjacent to the site of impaction was observed; in one case these changes were followed by progressive ischaemia of the retina.

\section{Case 1}

A man aged 62 who had enjoyed good health attended the eye clinic on 4 April 1964 complaining of sudden total loss of vision in the left eye three days previously; vision had returned gradually after two hours. A similar attack had occurred the next day.

* Consultant Eye Surgeon, Royal and City General Hospitals, Sheffield. t Consultant Ophthalmic Surgeon, King's Mill Hospital and Mansfield Hospital Group.

\section{Ocular Findings}

Corrected vision, $6 / 5$, N.5 each eye. The right eye was entirely normal.

In the left fundus a small white infarct of the retina was noted alongside the superior nasal artery close to the disc (I in Fig. 1) (Special Plate). A yellow-white bright glittering plaque was observed at the first bifurcation of the superior temporal branch of the central retinal artery (E in Fig. 1). There was no apparent interference with blood flow. Moderate digital pressure through the lower lid on to the globe caused the patch to flash, further pressure caused partial collapse of the central retinal artery and its juxtapapillary branches; at this moment the bright patch moved downwards on to the disc. Sudden release of pressure resulted in the patch shooting back to its original position. This observation, confirmed by several competent observers, was made with the patient both in the upright and in the supine position. Visual-field studies showed a small scotoma corresponding in position and size with the infarct $\mathrm{I}$. Three days later the plaque $\mathrm{E}$ in the left fundus was visible only when pressure was exerted on the globe. It could still be displaced towards the disc as described above.

On 10 June a new plaque appeared at the bifurcation of the left inferior papillary artery, $b$ in Fig. 2 (Special Plate). Three more plaques appeared in the left fundus over the next few months; they were located at various arteriolar bifurcations in the far periphery of the fundus and disappeared without sequels after a few weeks, as did embolus E, shown in Fig. 1. Meanwhile it was observed that the plaque located at the bifurcation $b$ of the inferior papillary artery appeared to be inducing an opaque change in the adjacent vessel wall. This change was first observed on $8 \mathrm{July}$, approximately one month after the appearance of the embolus. By 20 August the vessel walls were so opaque that the blood column and the

\section{LEGENDS TO SPECIAL PLATE}

Fig. 1.-Case 1. Extraneous pressure on the globe caused displacement of cholesterol embolus (E) to disc; release of pressure was followed by $\mathrm{E}$ shooting back to original position. A small infarct is shown (I). Pointer indicates bifurcation of inferior infarct is shown (b). Pointer indicates bifurcation of inferior papillary artery (b), which at this time

Fig. 2.-Case 1. Records lodgement of cholesterol embolus at b two months later. $(+10$. $)$

Fig. 3.-Case 1. Mural changes at and proximal to $b$ which began a few weeks after impaction and were maximal after two months. When the photograph was taken one year later the appearances were unchanged. $(\times 10$.

FIG. 4.-Case 2. Pointer is directed to embolus lodged at first bifurcation of inferior papillary artery. $(\times 10$.

Fig. 5.-Case 2. Vascular opacification proximal and distal to site of impaction. Cluster of white infarcts correspond with progress of vascular changes up to origin of a small arteriolar side branch $\left(a^{\prime}\right)$. $(\times 10$.

Fig. 6.-Further extension of vascular changes has occurred distally. Another arteriolar branch $\left(a^{\prime \prime}\right)$ is about to become involved. $(\times 10$. 
A. J. DARK AND S. N. RIZK: PROGRESSIVE FOCAL SCLEROSIS OF RETINAL ARTERIES
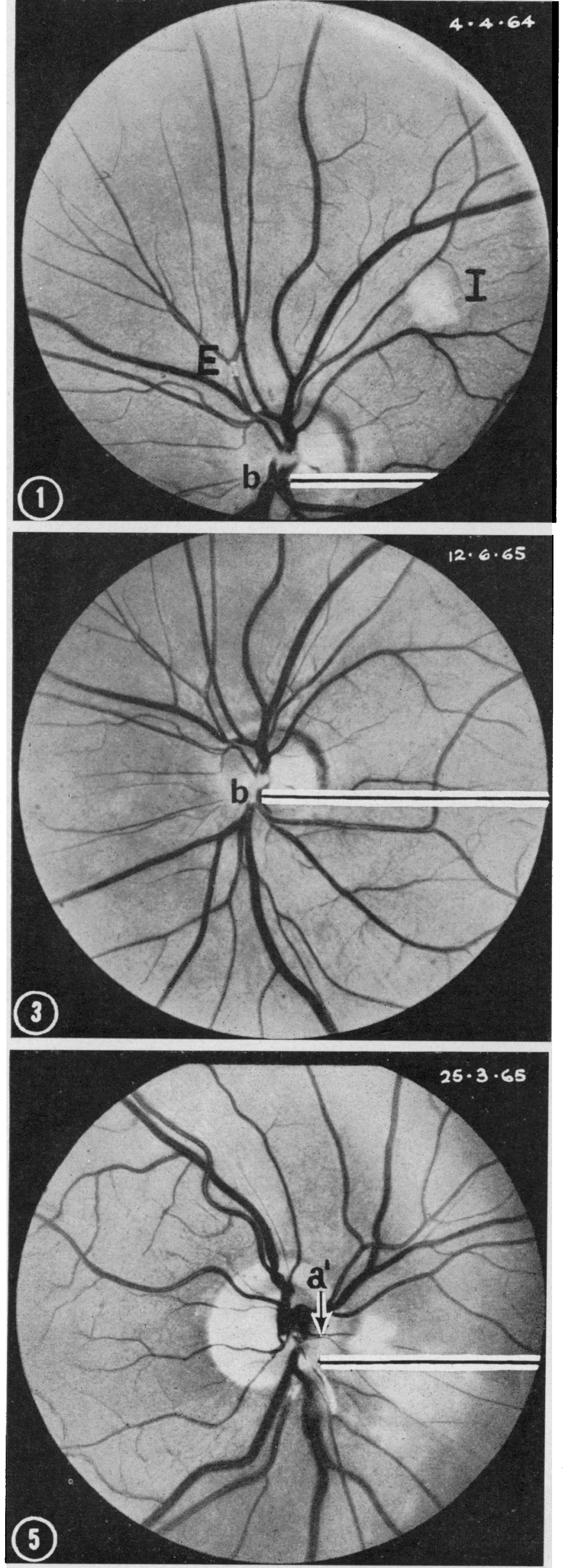
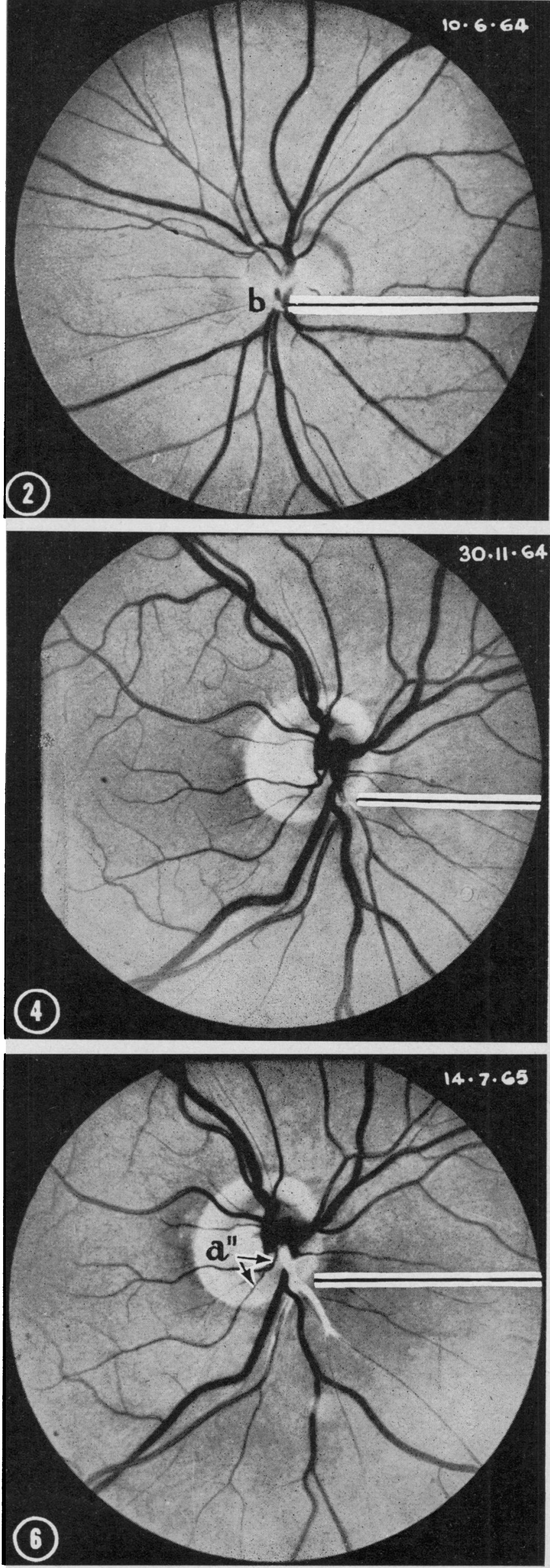

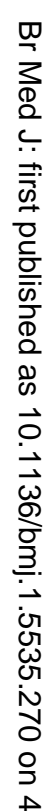

f

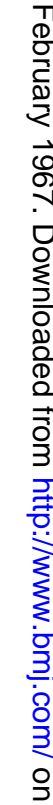

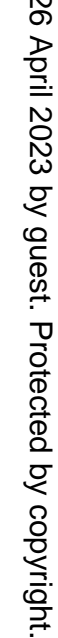


A. J. DARK AND S. N. RIZK: PROGRESSIVE FOCAL SCLEROSIS OF RETINAL ARTERIES

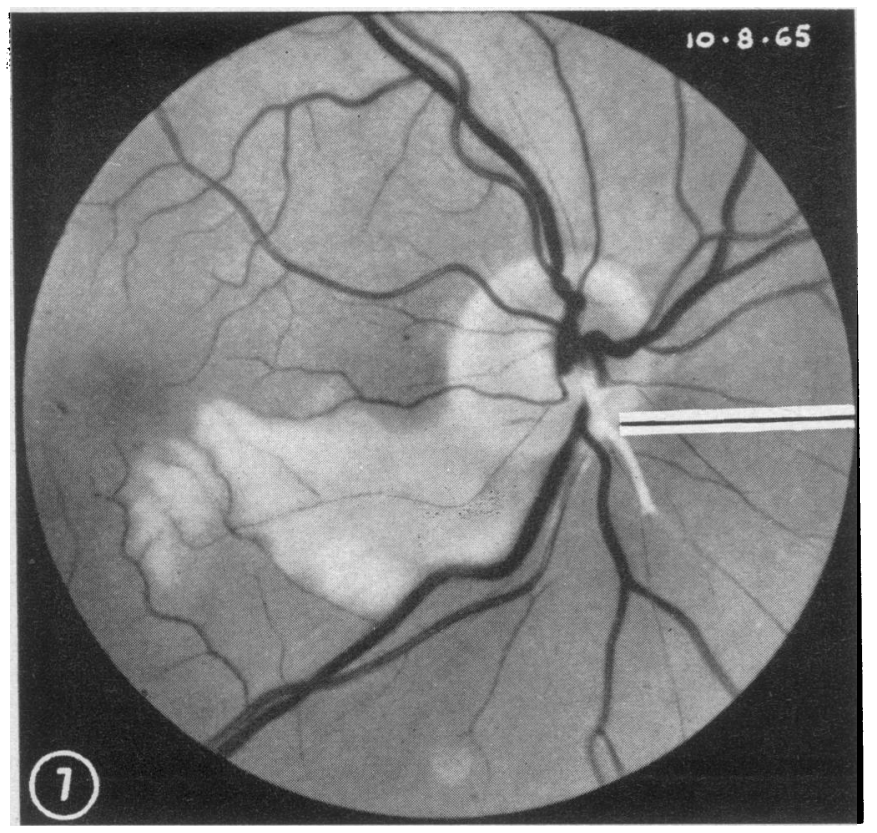

FIG. 7.- $a$ " has been occluded with subsequent extensive infarction of the retina. ( $\times 10$.

OTTO LJUNGBERG ET $A L .:$ MEDULLARY THYROID CARCINOMA AND PHAEOCHROMOCYTOMA

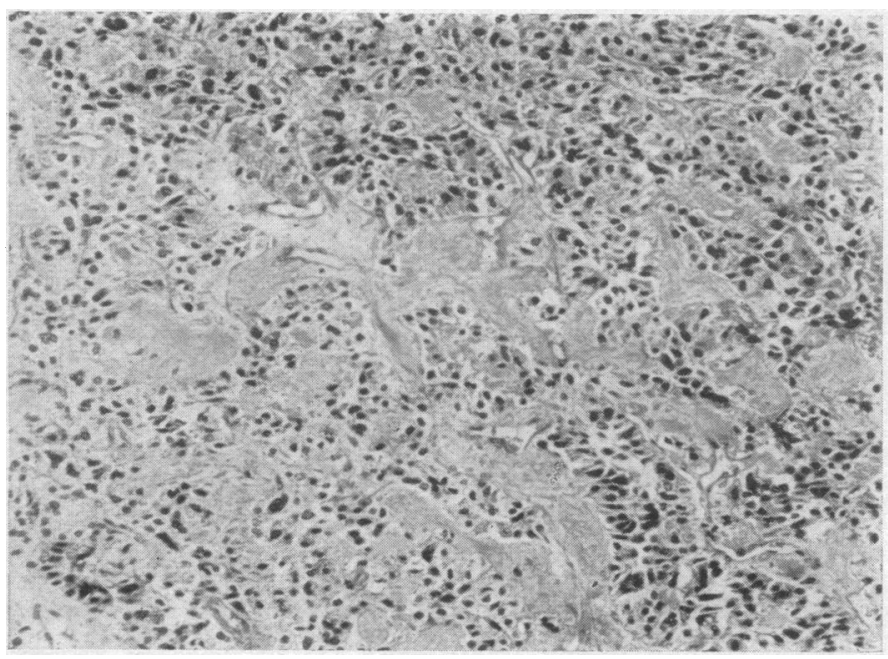

FIG. 1

Fig. 1.-Classical picture of medullary thyroid carcinoma (Case 7) with small irregular clusters of tumour cells in the stroma and a varying amount of amyloid. (Haematoxylin-erythrosin. $\times 125$. .)

Fig. 2.-Carcinoid-like picture of medullary thyroid carcinoma (Case 7) with large and small nests of small tumour cells poor in cytoplasm with a tendency to palisade arrangement of the cells in the margin. The stroma is sparse and the deposition of amyloid scanty. (van Gieson. $\times 78$.)

FIG. 3.-Spindle-cell picture of medullary thyroid carcinoma (Case 12) with elongated regular tumour cells crowded in whorls and streaks and with marked tendency to palisade formation: The stroma here is scanty and the deposition of amyloid insignificant. (Haematoxylin-erythrosin. $\times 100$.)

FIG. 4.-Section from right-sided phaeochromocytoma (Case 10). The picture agrees with that of a phaeochromocytoma with small alveolar formations of polygonal cells rich in cytoplasm in a delicate connectivetissue stroma. Specific green-staining of the cytoplasm in most of the cells. (Potassium bichromate and Giemsa staining. $\times 100$.)
OTTO LJUNGBERG ET $A L .:$ MEDULLARY THYROID CARCINOMA AND PHAEOCHROMOCYTOMA

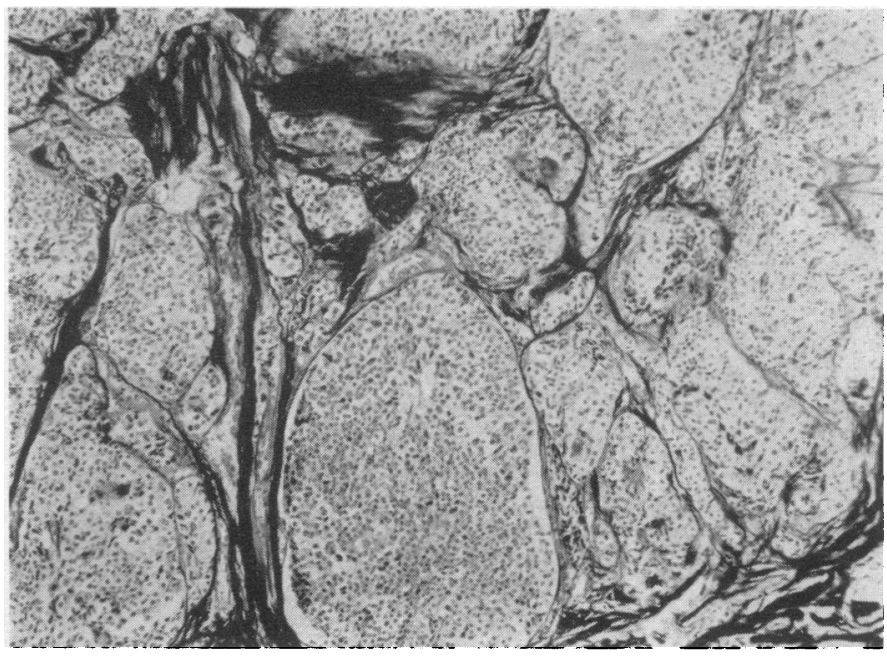

Fig. 2

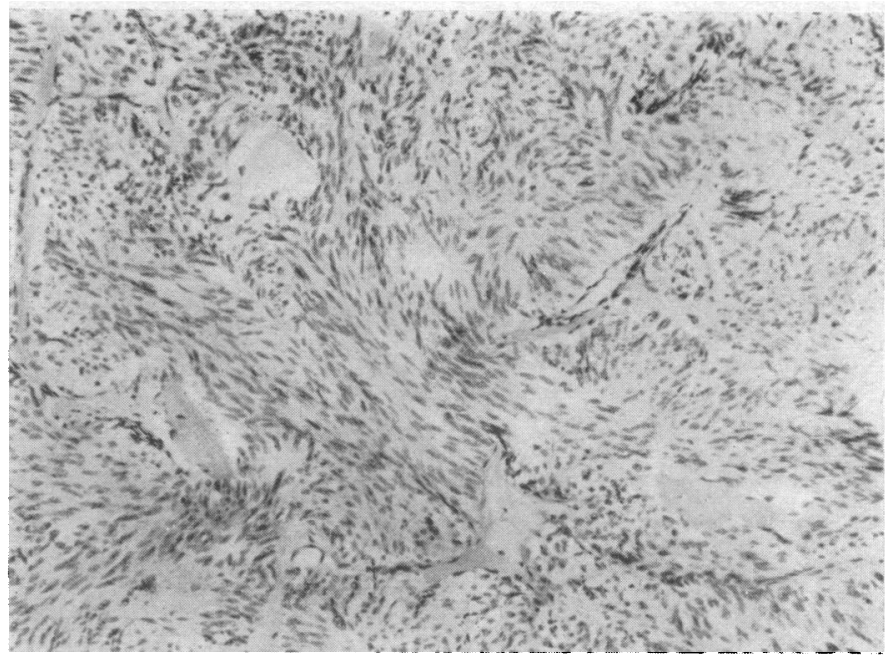

FIG. 3

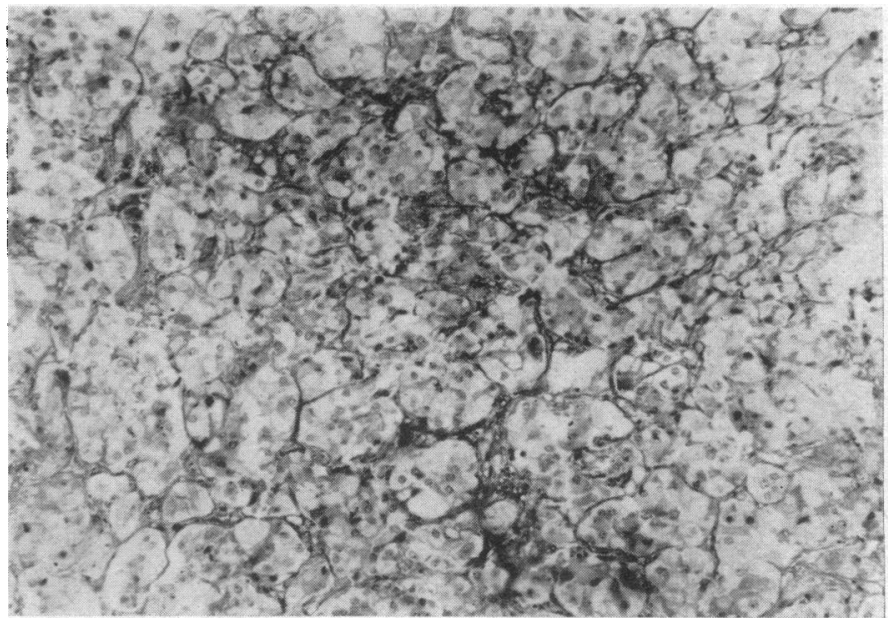

FIG. 4 
original plaque were no longer visible; moreover, the outlines of the vessel were by now ill-defined. The blood flow through this vessel was not apparently obstructed, and visual acuity, fundus appearances (Special Plate, Fig. 3), and fields of vision were unchanged 12 months later. Routine physical examination and investigations were normal.

\section{Case 2}

A man aged 62 attended the eye clinic on 30 November 1964 complaining of sudden loss of vision in the right eye. The loss of vision had been total and had lasted about one hour.

\section{Ocular Findings}

Corrected vision, 6/5, N.5 each eye. Positive findings were confined to the right fundus, in which three gleaming plaques were
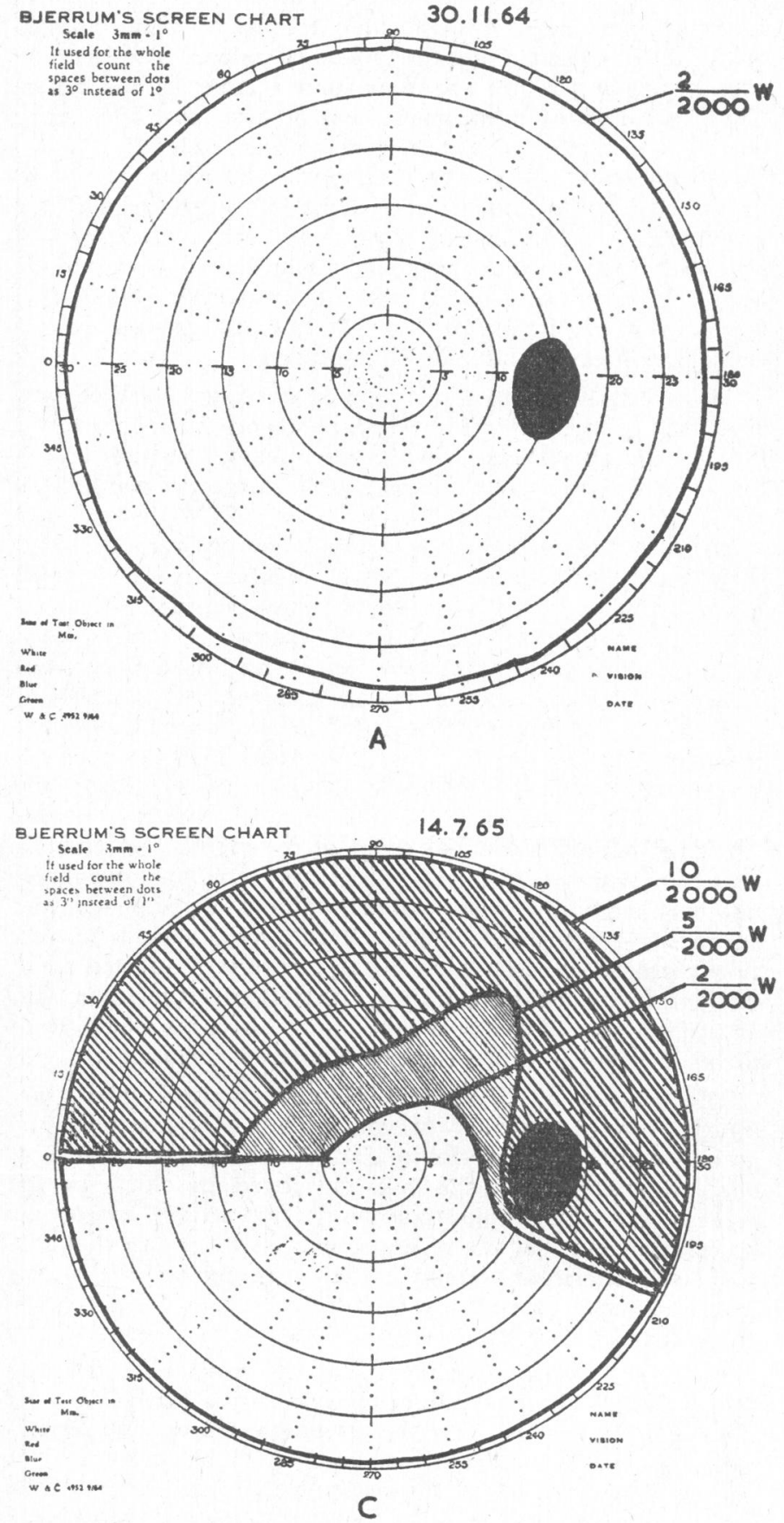

located at various bifurcation points of the central retinal artery. When digital pressure was exerted on the eye the plaques became brighter, flashing in heliograph fashion. One of the plaques was present at the bifurcation of the inferior papillary artery (Special Plate, Fig. 4). A second lay in the inferior temporal artery below the macula ; a small retinal infarct was present immediately distal to this site. A third plaque was found in the extreme retinal periphery. No visual-field defect was elicited (Fig. A).

A few days later the arterial wall adjacent to the plaque located at the bifurcation of the inferior papillary artery showed considerable opacification. This process continued at a slower rate for the next few months along the axis of the affected vessel both proximally and distally; at the same time the arterial column appeared to be undergoing progressive attenuation at the site of these changes.

Late in March 1965 a cluster of infarcts (cotton-wool patches), together with a few streak haemorrhages, appeared in the nervefibre layer alongside the inferonasal aspect of the disc (Special
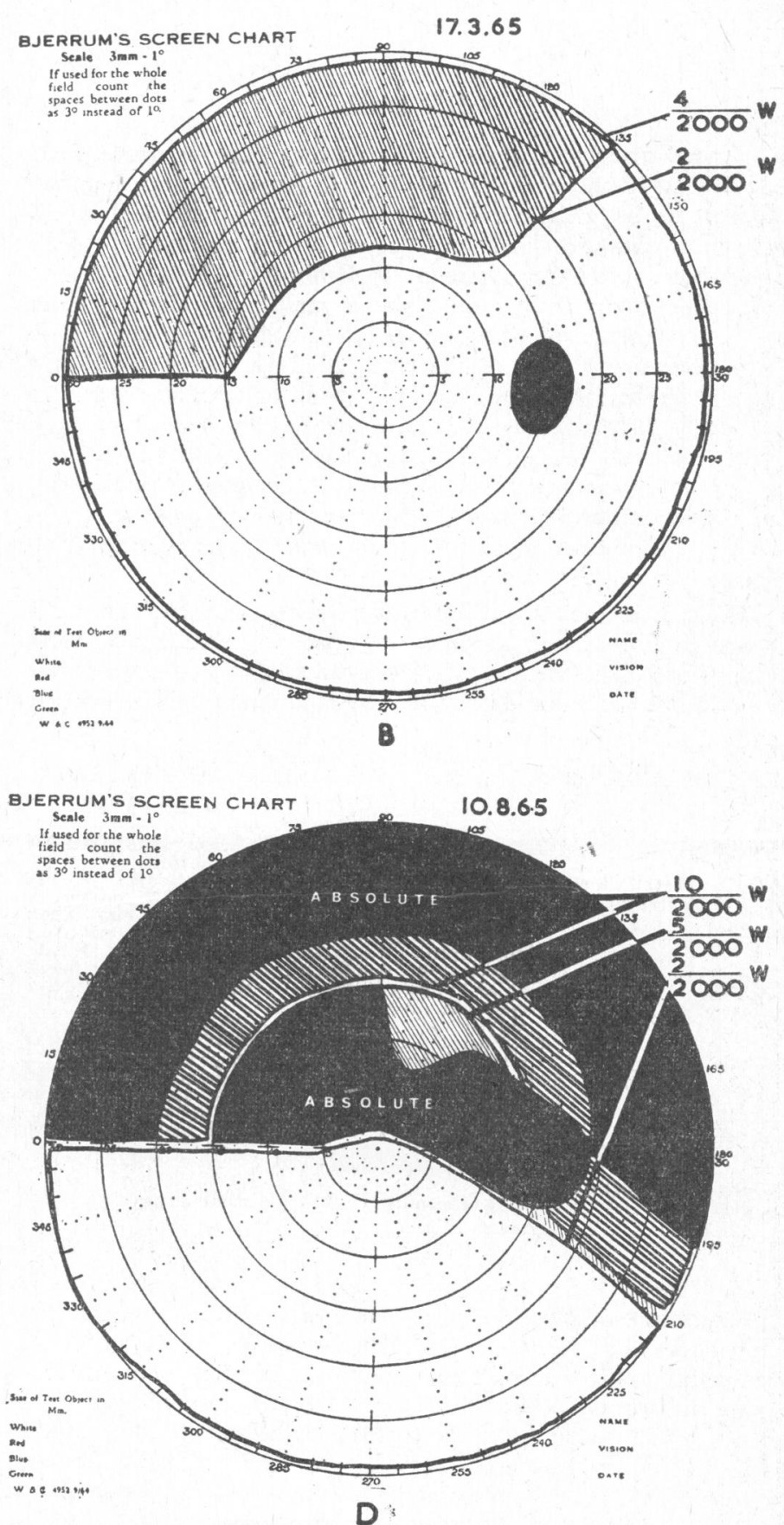

Figs. A.-D.- Case 2. Visual-field changes correspond with fundus appearances in Figs. 4 to 7 (Special Plate). Analysis of these changes indigressive ischaemia in the territory of the sclerosing inferior papillary artery with superadded acute abscilute field loss after successive D infarcts produced by occlusion of arterioles $a^{\prime}$ and $a^{\prime \prime}$. 
Plate, Fig. 5). This area of the retina is supplied by an arteriole $a^{\prime}$ (Fig. 5) taking origin from the inferior papillary artery, and the sudden appearance of the infarcts coincided with the proximal extension of the mural changes up to the point of origin of this vessel. A few weeks later the cotton-wool patches were no longer evident. The mural changes continued to progress; their appearances on 14 July 1965 are recorded in Fig. 6 (Special Plate). By 10 August further proximal extension of the changes in the inferior papillary artery had involved yet another arteriolar branch of this artery $a^{\prime \prime}$; this and the resultant area of infarction are seen in Fig. 7 (Special Plate). From 17 March 1965 onwards visual-field studies, which had previously revealed no defects even to small coloured targets, now showed a progressive field loss (Figs. A, B, C, and D). Analysis of these field defects indicates chronic progressive loss of function in the territory supplied by the inferior papillary artery, with the superimposed areas of acute absolute loss corresponding with infarcts recorded in Figs. 5 and 7.

Apart from the ocular findings the patient was found to have mild diabetes mellitus. The results of other investigations were all normal.

\section{Discussion}

Retinal arterial plaques in the present patients correspond closely with those described by Hollenhorst, and likewise appear to be cholesterol emboli, though histological proof was not obtained. In both cases serum cholesterol levels were normal, as they were in Hollenhorst's (1961) series of patients. Apart from mild diabetes mellitus in Case 2, there was no evidence of systemic disease in either patient. It is aiso noteworthy that angiograms failed to reveal significant disease in the ipsilateral internal carotid arteries in these two patients. Bright plaques in retinal arteries have, until recently, been regarded as a local arteriosclerotic manifestation and generally considered to be deposits of cholesterol developing in atheromatous plaques. It now seems that most of them, if not all, are in fact embolic in nature, originating from eroded atheromatous sites.

Hollenhorst has given important indirect evidence for their embolic nature; thus he noted that pressure on the eye would sometimes cause the patches to fragment and pass out of view or to distal points of impaction. Swan, quoted by Hollenhorst

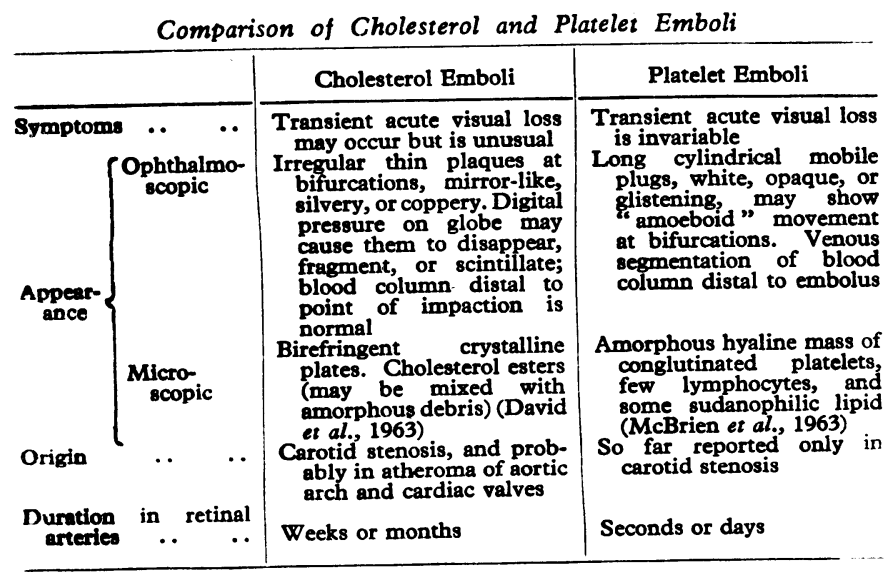

et al. (1962), has apparently taken cinephotographs of such plaques in motion. Injection of human atheromatous material into dogs and monkeys (Hollenhorst et al., 1962) produced gleaming retinal emboli resembling those seen in man; they were subsequently identified as thin plates of cholesterol. A fatal human case of atheromatous embolism of the middle cerebral artery provided David et al. (1963) with the opportunity for microscopical examination of 12 bright plaques which suddenly appeared in the ipsilateral retinal arteries several hours before death. By using histochemical and microchemical techniques on wholemount preparations of retina these authors were able to demonstrate that the plaques were composed of birefringent crystals containing cholesterol esters. Detailed histological examination on sectioned material was not apparently undertaken.

In our Case 1 a plaque " $\mathrm{E}$ " was displaced by pressure on the globe towards the disc against the blood flow over a considerable segment of the affected blood vessel and release of pressure allowed it to shoot back to its original site of impaction. This repeatable phenomenon in an otherwise apparently healthy artery provides further evidence for the intraluminar situation of the plaques and therefore for their embolic nature. Retinal emboli in cases of internal carotid artery stenosis described by Fisher (1952), Russell (1961), and McBrien et al. (1963) appear to be of a different nature, being largely composed of conglutinated platelets. The Table summarizes what appear to be important differences in these two types of embolic phenomena. The two cases presented here were directed to the eye clinic because of their common complaint of transient monocular loss of vision. The cause of such visual loss is not apparent from a study of the fundi. Three possibilities occur: (1) these attacks may have been caused by impaction of platelet material independently ; (2) the cholesterol emboli may have initially caused sufficient obstruction of the central retinal artery at its bifurcation to produce brief retinal ischaemia, this being relieved by subsequent fragmentation ; or (3) the original embolus may have contained both crystalloid and other thrombus material, the latter gradually dissociating from the crystals and passing to the retinal periphery and finally disappearing.

In modern literature the subject of cholesterol emboli was first dealt with by Flory (1945), who reported its occurrence in nine necropsy cases showing atheromatous erosion of the aorta. Presumed emboli were found in arteries varying in size from 50 to $900 \mu$ in the pancreas, spleen, thyroid, and kidney. Microscopically the arterial lumina were occluded with hyperplastic intimae in which acicular spaces (site of dissolved-out cholesterol crystals) were present; alongside these spaces a few giant cells were noted. Handler (1956) extended these observations by describing seven more cases having segmental atrophy of the renal cortex (focal encephalomalacia and diffuse cerebral cortical atrophy were also present in some of these cases). Handler noted that there was a surprising paucity of evidence for blood-clot formation in the occluded vessels, a finding he felt might be due to the fact that total occlusion did not occur at the moment of embolic impaction nor even in the early reactive granulomatous phase, but resulted only when intimal fibrous hyperplasia had advanced to a marked extent. If this hypothesis is correct slowly progressive ischaemia in the territory of affected small end-arteries would be expected rather than acute infarction, and indeed the histological findings in his cases would seem to be in keeping with progressive arterial occlusion.

The studies of Flory and Handler and of others are fraught with a difficulty of which these authors were well aware-namely, the possibility that the cholesterol crystals at sites of arterial occlusion had developed in situ. In the present ophthalmoscopic study the development of progressive mural change at the site of impaction has been observed as a sequel to cholesterol embolization. Moreover, the slowly progressive field loss seen in Case 2 demonstrates that gradual focal ischaemia may indeed result from such changes.

In Case 1 a slow opacification of the vessel wall (diameter $90 \mu$ ) was first noticed ophthalmoscopically a few weeks after the appearance of the embolus. It progressed to total opacification but without apparent obstruction to blood flow. One year later no field defect was discernible and the calibre of the artery distal to the point of impact was unaltered. In Case 2 a vessel of similar diameter showed early opacification at the site of impaction when the patient was first seen. These changes 
in Case 2 were more rapid and extensive and subsequently produced serious loss of visual field. These field changes were of two distinct types: (1) an insidious progressive loss corresponding with gradually increasing ischaemia in the retinal territory supplied by the diseased vessel, and (2) additional acute absolute loss produced by successive involvement and occlusion of the arteriolar branch of this vessel with resultant retinal infarcts. Vascular changes described as "sheathing" occurring after cholesterol emboli were noted by Balla et al. (1964). The same authors mention that this reaction tended to occur in the larger retinal arteries, and the present observations seem to bear this out; that is to say, the mural changes were seen in small retinal arteries rather than in arterioles. This observation also broadly agrees with the findings of the authors mentioned above, who studied necropsy material from a variety of organs, excluding the eye.

The origin of cholesterol emboli in the present cases has not been determined. The ipsilateral carotid systems showed no filling defects distal to the site of angiography puncture. The possibility of atheroma proximal to this point has not been eliminated in these two patients, cardiac disease was not evident clinically, electrocardiograms were normal, and straight $x$-ray films of the mediastina did not reveal calcification in the heart or the great vessels. It is of course possible that erosion of atheromatous plaques in the internal carotid or other great vessels may occur in cases showing no angiographic defect or $x$-ray opacities due to calcification.

Transient unilateral loss of vision which is not obviously due to carotid stenosis (that is, its occurrence in patients in whom the difference in ophthalmodynamometry readings on the two sides is repeatedly less than $15 \%$ ) has been categorized from an aetiological standpoint by Cogan (1961). In one group he found bright refractile plaques in the ipsilateral retinal arteries, and transient loss of vision in these cases was thought to be due to local arteriosclerotic change. It now seems likely that these cases should be grouped with the present Cases 1 and 2, in which the bright plaques have been identified as cholesterol emboli and which have been demarcated from those with angiographically demonstrable occlusive carotid disease.

\section{Summary}

Two patients presenting with transient monocular loss of vision were found to have cholesterol embolization of the ips1lateral retinal arteries. There was no angiographic evidence of ipsilateral carotid occlusive disease in either patient.

A review of the literature equating bright plaques in retinal arteries with cholesterol emboli is given. Present observations have provided further evidence in favour of the embolic nature of these plaques.

Serial fundus photographs record progressive sclerotic changes after the impaction of cholesterol emboli in what were previously apparently healthy retinal arteries.

In Case 1 these changes were slow in evolving and were not associated with retinal ischaemia one and a half years later. In Case 2 similar but more rapid vascular sclerosis produced chronic retinal ischaemia in the territory of the affected artery with superadded successive acute infarction. Visual-field studies have closely corroborated these findings.

It is a pleasure to acknowledge the assistance given by Dr. Keith Brammar, who was responsible for taking the fundus photographs. We are also grateful to Dr. J. P. P. Bradshaw, consultant neurologist at the Royal Hospital, Sheffield, and to Professor F. Jacoby, Director of Subdepartment of Histology, University of Cardiff, South Wales, for their valuable comments during the preparation of the manuscript.

\section{REFERENCES}

Balla, J. I., Howat, J M. L., and Walton, J. N. (1964). F. Neurol. Neura surg. Psychiat.; 27, 144.

Cogan, D. G. (1961). Arch. Ophthal., 66, 180.

David, N. J., Klintworth, G. K., Friedberg, S. J., and Dillon, M. (1963). Neurology (Minneap.), 13, 708.

Fisher, M. (1952). Arch. Ophthal., 47, 167.

Fisher, M. (1952). Arch. Ophthal., 47, 167.

Flory, C. M. (1945). Amer. F. Path., 21, 549.

Hollenhorst, R. W. (1961). Trans. Amer. ophthal. Soc., 59, 252. Hollenhorst, R. W. (1962). Trans. Amer. Acad. Ophthal. Otolaryng., 66, 166.

- Lensink, E. R., and Whisnant, J. P. (1962). Trans. Amer. ophthal Soc., 60, 316 .

McBrien, D. J., Bradley, R. D., and Ashton, N. (1963). Lancet, 1, 697

Russell, R. W. R. (1961). Ibid., 2, 1422.

Sayre, G. P., and Campbell, D. C. (1959). Arch. intern. Med., 103, 799.

\title{
Correlation of Chromosome Counts with Histological Appearances and Prognosis in Transitional-cell Carcinoma of Bladder
}

\author{
DAVID LAMB,* M.B., B.S., B.SC.
}

Brit. sned. 7., 1967, 1, 273-277

The study of chromosome changes in malignant tumours has become an important aspect of cancer research in recent years. With the exception of some leukaemias, all primary human malignant tumours examined without prior cell culture have had abnormal chromosome sets (Spriggs, 1964), but little correlation has emerged between the chromosome changes and the histological appearances.

Atkin and Richards (1962) and Atkin (1964), using microspectrophotometric measurements of nuclear deoxyribose nucleic acid (D.N.A.), have shown that high ploidy in squamous carcinoma of the cervix is associated with a good prognosis, but not with any particular histological appearance. In adenocarcinomas of the uterine body, however, high ploidy was found to be associated with a poorly differentiated histological pattern but not with prognosis.

- Research Assistant, Institute of Diseases of the Chest, London.
In none of the large published series of chromosome studies on human material (Makino et al., 1959, 1964 ; Ishihara et al., 1963 ; Spriggs et al., 1962b ; Hauschka, 1963) have these findings been confirmed or similar findings reported for tumour at other sites.

Shigematsu (1965) has studied the chromosomes in a series of transitional-cell carcinomas of the bladder. He found a relation between the levels of chromosome count and the histological picture.

It was decided to study the relation of chromosome number to the pathological findings in a series of transitional-cell carcinomas of the bladder. Transitional-cell carcinoma of the bladder was chosen because there is a well-recognized relation between the histological grade of malignancy and the presence and depth of invasion, and between these two factors and prognosis. 